\title{
The influence of packing dimension on the effective thermal properties of doubly-periodic composites by using super hybrid finite body elements
}

\author{
Hui Wang ${ }^{1,2}$, Yong-Peng Lei ${ }^{1}$, Zhao-Ran Xiao ${ }^{3}$ \& Li Chen ${ }^{1}$ \\ ${ }^{I}$ Department of Engineering Mechanics, \\ Henan University of Technology, China \\ ${ }^{2}$ Institute of Scientific and Engineering Computation, \\ Henan University of Technology, China \\ ${ }^{3}$ College of Civil Engineering and Architecture, \\ Henan University of Technology, China
}

\begin{abstract}
A super polygonal hybrid element is developed for investigating the effects of the packing dimension on effective thermal conductivities of composites containing a doubly periodic array of circular inclusions. In the analysis, a rectangular representative volume element (RVE) is employed to represent the packing dimension of the periodic microstructure and then is solved using a super hybrid finite element in which the fundamental solutions are taken as internal interpolation functions. The fundamental solutions used to satisfy both the heat transfer governing equation and the interfacial continuity between the inclusion and the surrounding matrix. The use of fundamental solutions makes the functional of stiffness matrix including boundary integrals only. As a result, significant efforts on mesh generation can be saved, and mesh reduction as well as high solution accuracy can be achieved. Finally, numerical examples are considered to investigate the role of the packing dimension of the RVE.

Keywords: doubly periodic composites, packing dimension, representative volume element, effective thermal conductivity, super hybrid finite element.
\end{abstract}




\section{Introduction}

The determination of effective thermal properties of composites is of importance in the design of structures with composite materials. One of the important factors that affect the effective thermal properties is the microstructure of the composites. The microstructure here means the shape, size, material properties and spatial distribution of the reinforcing inclusions. During the years, various numerical techniques have been developed for revealing the variation of effective thermal properties of composites due to microstructure.

In particular, considerable attention was paid to the calculation of effective thermal conductivity of composites using the finite element method (FEM) [1-3]. For example, Islam and Pramila [1] studied the thermal conductivity of periodic fiber reinforced composites using FEM and considered four different boundary conditions on the boundary of RVE. They found that the case of prescribed temperature conditions can produce most accurate results up to a high volume fraction of fiber. Using translational symmetry transformations, Li [2] investigated two typical idealised packing systems for unidirectional fibre reinforced composites, viz. square and hexagonal ones, by FEM. Li et al. [3] employed the finite element technique implemented by the commercial software ABAQUS to solve the hexagon RVE for determining the effective thermal conductivity of woven fabric composites. Usually, the implementation of FEM to simulate fiber or particle filled composites with high inclusion volume fraction results in an extremely large number of elements in the vicinity of the material interface in order to obtain an acceptable accuracy, due to restriction of element connectivity and the requirement of an appropriate value of element aspect ratio. As a numerical technique different to the traditional FEM, the boundary element method (BEM) was developed to evaluate effective thermal properties of composites with significant characteristics of boundary line/face discretization (instead of face/volume discretization in FEM) for each material constituent [46]. Recently, the boundary-type meshless method of fundamental solutions (MFS) was developed for determination of effective thermal conductivity of unidirectional composites with linearly temperature dependent conductivity of constituents [7]. However, in the analysis of composite materials, the BEM or MFS requires additional coupling equations to meet the interfacial continuity between adjacent material phases, beside the boundary integral equation for each material phase. As a result, the computational complex increases. Moreover, to investigate the effect of volume fraction of inclusion on the properties of composite structures, both FEM and BEM need a remeshing procedure as the volume fraction of inclusion changes.

As an alternative to the element-based methods like the FEM and the BEM, a hybrid finite element formulation based on a fundamental solution kernel (HFSFEM) was presented by Wang and Qin for the two-dimensional heat conduction problem $[8,9]$, the linear elasticity with circular or elliptical holes $[10,11]$, the functionally graded structures $[12,13]$, and the nonlinear potential problems [14] in the past several years. In this paper, the HFS-FEM was extended to determine the influence of packing size on the transverse effective thermal conductivities of 
composites with doubly periodic-distributed fibres in which the fibre is regularly and unidirectionally aligned. Under the doubly periodic assumption, the RVE is modeled as a rectangular domain with a single circular inclusion and surrounding matrix phase, and proper periodic boundary conditions are applied for the effect of the surrounding matrix material. Subsequently, the numerical thermal response in the chosen microstructure is solved using a developed super polygonal hybrid element presented in the HFS-FEM, which is formulated by combining a modified energy functional, element interior temperature and heat flux fields based on the superposition of special fundamental solutions analytically satisfying the governing differential equations and the interfacial continuity of temperature and heat flux between the matrix and the inclusion, and element boundary temperature field by shape function interpolation. With numerical results, the thermal properties of the equivalent orthotropic medium can be predicted. In the study, a super polygonal element with multiple edges and nodes is designed to enclose the inclusion and all integrals associated with the present element are nonsingular boundary integrals. Thus, these features of the present super hybrid element enable us to avoid mesh division within the inclusion and change the inclusion volume fraction arbitrarily without mesh regeneration during the RVE thermal analysis.

\section{Microstructure model}

For a two-dimensional doubly periodic composite system, in which the distribution of inclusions is uniform and symmetric, the microstructure model usually consists of a unit cell which encloses the smallest periodic repeat volume in the bulk composite materials and should be able to construct the whole composite by repeated packing of the unit cell in two directions of coordinate axes. Let $d$ be the diameter of the circular inclusion and $l, h$ be the packing spacings in the horizontal and vertical directions, respectively. The $x_{1}$ - and $x_{2}$ axes are taken with their origin at the center of one of the inclusions considered. Figure 1 illustrates the schematic of the composite with a doubly periodic array of circular inclusions and the corresponding rectangular unit cell, which is also named as the RVE. The periodic unit cell consists of two different regions, i.e., matrix and circular inclusion, respectively. Each region has its own homogeneous and isotropic thermal conductivity and a perfect bond is assumed between these two regions. In this study, the different packing sizes (or RVE sizes) along the two coordinate directions are assumed for investigating packing pattern effect.

To derive the microstructure mathematical model, we assume that no thermal contact resistance between the inclusion and the matrix exists. According to the heat conduction constitutive law (Fourier law), the heat flux components can be written in terms of temperature gradient as

$$
q_{i}=-k \frac{\partial T}{\partial x_{i}}
$$


where $T$ is the temperature field and $k$ represents the thermal conductivity of the inclusion or matrix. In this study, the superscripts $M$ and I stand for quantities associated with the matrix and the inclusion, respectively.

A heat transfer analysis of the chosen microstructure satisfying the feature of double packing requires appropriate periodic temperature boundary conditions, which are prescribed along all the sides of the chosen RVE. For a regular double packing in the $x_{1}$ and $x_{2}$ directions, the symmetries, which will be used to obtain the coordinate relations between a unit cell and its image, are the translations along the $x_{1}$ and $x_{2}$ axes, as shown in Figure 1. Under these translational symmetries, any point $\tilde{P}\left(\tilde{x}_{1}, \tilde{x}_{2}\right)$ in an arbitrary cell can be mapped to its image $P\left(x_{1}, x_{2}\right)$ and their coordinates are related as follows [2]

$$
\tilde{x}_{1}=x_{1}+2 j l, \quad \tilde{x}_{2}=x_{2}+2 k h
$$

where $j, k=0, \pm 1, \pm 2, \cdots$ are the numbers of cells by which $\tilde{P}\left(\tilde{x}_{1}, \tilde{x}_{2}\right)$ is away from $P\left(x_{1}, x_{2}\right)$ in the $x_{1}$ and $x_{2}$ directions, respectively.

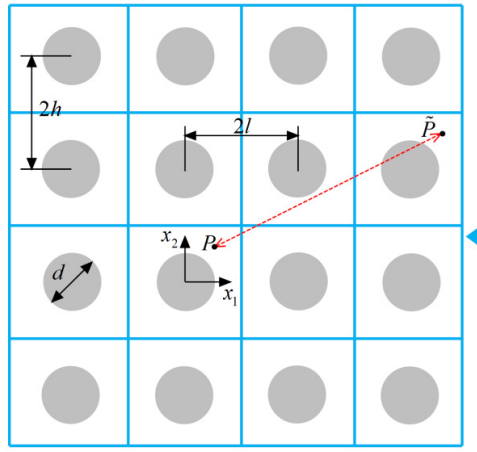

(a) Composites with doubly-perodic array of inclusions

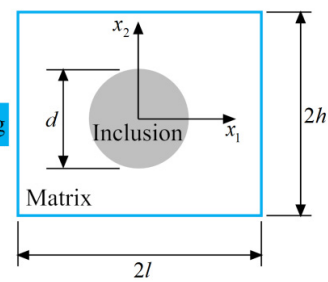

(b) Rectangular RVE

Figure 1: Schematic of periodic double-array composite and representative volume element (RVE).

In particular, for the chosen cell, the two transformations can be given by setting $j$ and $k$ to the two paired sides as follows:

$$
\begin{array}{ll}
j=1, k=0 & \text { for paired sides } x_{1}= \pm l \\
j=0, k=1 & \text { for paired sides } x_{2}= \pm h
\end{array}
$$

Subsequently, the induced temperature difference between $P\left(x_{1}, x_{2}\right)$ and $\tilde{P}\left(\tilde{x}_{1}, \tilde{x}_{2}\right)$ in different cells can be written as

$$
\tilde{T}-T=\left(\tilde{x}_{1}-x_{1}\right) \frac{\partial T^{0}}{\partial x_{1}}+\left(\tilde{x}_{2}-x_{2}\right) \frac{\partial T^{0}}{\partial x_{2}}
$$

in which $T^{0}$ is the macroscopic temperature field.

Substituting Eqs. (2) and (3) into (4), one has the following periodic temperature conditions on the two paired sides 


$$
\left.T\right|_{x_{1}=l}-\left.T\right|_{x_{1}=-l}=2 l \frac{\partial T^{0}}{\partial x_{1}},\left.\quad T\right|_{x_{2}=h}-\left.T\right|_{x_{2}=-h}=2 h \frac{\partial T^{0}}{\partial x_{2}}
$$

In this paper, a medium containing doubly periodic inclusions is considered as a homogeneous orthotropic medium due to the rectangular packing $[1,2]$, and the corresponding Fourier's law for orthotropic heat conduction is given by

$$
q_{1}^{0}=-k_{1}^{\mathrm{C}} \frac{\partial T^{0}}{\partial x_{1}}, \quad q_{2}^{0}=-k_{2}^{\mathrm{C}} \frac{\partial T^{0}}{\partial x_{2}}
$$

In order to determine the effective thermal property $k_{1}^{C}$ of the composite, the proper boundary conditions should be prescribed to the chosen RVE for solving the heat conduction problem in the RVE. According to the work of Islam and Pramila [1], the periodicity achieved by the following prescribed temperature boundary conditions on the vertical boundaries of RVE that can produce most accurate results up to a relatively high fiber volume fraction

$$
T\left(-l, x_{2}\right)=T_{1}, \quad T\left(l, x_{2}\right)=T_{2}
$$

where $T_{1}$ and $T_{2}$ are the constant temperature applied on the vertical boundaries of the RVE. In addition, the upper and lower boundaries of the cell are assumed to be insulating, i.e. $q_{2}\left(x_{1}, \pm h\right)=0$.

Under the boundary conditions defined above, the heat transfer in the RVE can be numerically solved and then the average heat flux component in the $x_{1}$-direction on the isothermal boundary can be evaluated by a simple integral

$$
q_{1}^{0}=\frac{1}{2 h} \int_{-h}^{h} q_{1}\left(l, x_{2}\right) \mathrm{d} x_{2}
$$

which can easily be calculated using the trapezoidal rule of numerical integration.

Simultaneously, the temperature gradient in the $x_{1}$-direction can be evaluated by the periodic temperature conditions (5) as

$$
\partial T^{0} / \partial x_{1}=\left(T_{2}-T_{1}\right) / 2 l
$$

Thus, substituting Eq. (9) into the first expression of Eq. (6) yields

$$
k_{1}^{\mathrm{C}}=-\left(q_{1}^{0} \cdot 2 l\right) /\left(T_{2}-T_{1}\right)
$$

Similarly, when the following temperature and heat flux boundary conditions are applied on the boundaries of the RVE

$$
T\left(x_{1}, h\right)=T_{2}, \quad T\left(x_{1},-h\right)=T_{1}, \quad q_{1}\left( \pm l_{1}, x_{2}\right)=0
$$

we have

$$
k_{2}^{\mathrm{C}}=-\left(q_{2}^{0} \cdot 2 h\right) /\left(T_{2}-T_{1}\right)
$$

with

$$
q_{2}^{0}=\frac{1}{2 l} \int_{-l}^{l} q_{2}\left(x_{1}, h\right) \mathrm{d} x_{1}
$$




\section{Formulation of the super element}

To find apparent thermal properties of the composites defined above, one must solve the corresponding boundary value problems (BVP) described by the heat transfer in the rectangular RVE with proper boundary conditions. In this study, the hybrid finite element formulation initially established for heat transfer simulation is extended to obtain the effective thermal properties of the doubly periodic composite and a super polygonal element with multiple sides and nodes is constructed to model the chosen RVE. In Figure 2, a typical super polygonal element with 12 sides and 24 nodes is shown as an example.

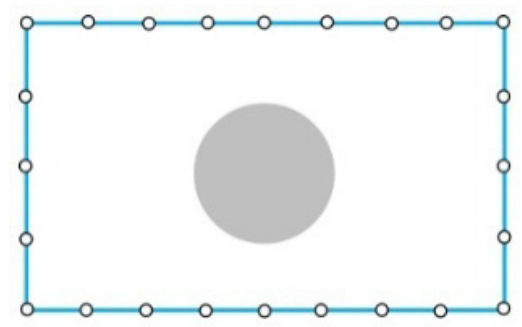

Figure 2: $\quad$ Illustration of the present super hybrid finite element.

Let $\Omega_{e}$ be the overall element domain consisting of matrix subdomain $\Omega^{\mathrm{M}}$ and inclusion subdomain $\Omega^{\mathrm{I}}$, and $\Gamma_{e}$ be its boundary. For the heat transfer problem in the RVE under consideration, the following hybrid functional is written as $[8,9]$

$$
\Pi_{e}=-\frac{1}{2} \int_{\Omega_{e}} k\left(\frac{\partial^{2} T}{\partial x_{1}^{2}}+\frac{\partial^{2} T}{\partial x_{2}^{2}}\right) \mathrm{d} \Omega-\int_{\Gamma_{q e}} \bar{q} \tilde{u} \mathrm{~d} \Gamma+\int_{\Gamma_{e}} q_{n}(\tilde{u}-u) \mathrm{d} \Gamma
$$

in which $T$ is the element interior temperature field to be assumed to naturally satisfy the governing equation of heat transfer in the two material phases and interfacial conditions between them, and $\tilde{T}$ is the element boundary temperature field which is introduced to enforce the conformity on the field variable between adjacent elements. $q_{n}=q_{1} n_{1}+q_{2} n_{2}$ and $\bar{q}$ are the normal heat flux and its specified value on the boundary $\Gamma_{q e}$, respectively. $n_{i}(i=1,2)$ are components of outward unit normal to the boundary.

Applying the divergence theorem to the functional (14) yields

$$
\Pi_{e}=-\frac{1}{2} \int_{\Gamma_{e}} q_{n} T \mathrm{~d} \Gamma-\int_{\Gamma_{q e}} \bar{q} \tilde{T} \mathrm{~d} \Gamma+\int_{\Gamma_{e}} q_{n} \tilde{T} \mathrm{~d} \Gamma
$$

In the formulation of the present hybrid finite element, the interior temperature and the corresponding normal heat flux in Eq. (15) can be given by [8]

$$
T=\sum_{j=1}^{n_{s}} G\left(z, z_{s j}\right) c_{j}=\mathbf{N c}, \quad q_{n}=-k \frac{\partial T}{\partial n}=\mathbf{Q c}
$$


where $G\left(z, z_{s j}\right)$ is the fundamental solution or Green's function associated with the inclusion, $z=x_{1}+x_{2} i$ and $z_{s j}=x_{1 j}^{s}+x_{2 j}^{s} i$ are the field point and source point expressed in the complex space, respectively, and $c_{j}\left(j=1,2, \cdots, n_{s}\right)$ denotes the unknown coefficient. $\mathbf{N}, \mathbf{G}$ and $\mathbf{c}$ in Eq. (16) are respectively the corresponding matrix or vector forms of approximation kernels or coefficients.

Here, to construct the super element, the special Green's functions analytically satisfying the governing differential equations corresponding to the inclusion and matrix phases and the interfacial continuity between them are employed $[9,15]$

$$
G\left(z, z_{s j}\right)=-\frac{1}{2 \pi k^{\mathrm{M}}}\left\{\operatorname{Re}\left[\ln \left(z-z_{s j}\right)\right]+\frac{k^{\mathrm{M}}-k^{\mathrm{I}}}{k^{\mathrm{M}}+k^{\mathrm{I}}} \operatorname{Re}\left[\ln \left(\frac{R^{2}}{z}-\bar{z}_{s j}\right)\right]\right\}
$$

for $z \in \Omega^{\mathrm{M}}$ and

$$
G\left(z, z_{s j}\right)=-\frac{1}{\left(k^{\mathrm{M}}+k^{\mathrm{I}}\right) \pi} \operatorname{Re}\left[\ln \left(z-z_{s j}\right)\right]
$$

for $z \in \Omega^{\mathrm{I}}$.

Typically, setting $k^{\mathrm{I}}=0$ or $k^{\mathrm{I}}=\infty$ in Eq. (17), one can obtain the special Green's functions respectively related to the cases of circular hole and superconductor. Here it is necessary to mention that for the case of the superconductor, Eq. (18) reduces to $G\left(z, z_{s j}\right)=0$, which means that the temperature distribution within the inclusion is uniform.

The independent element boundary temperature field $\tilde{T}$ appearing in Eq. (15) is defined in terms of nodal temperature vector $\mathbf{d}$ as

$$
\tilde{T}=\tilde{\mathbf{N}} \mathbf{d}
$$

where $\tilde{\mathbf{N}}$ represents the conventional interpolating shape function commonly used in the FEM or BEM.

Substituting Eqs. (16) and (19) into the functional (15) produces

$$
\Pi_{e}=-\frac{1}{2} \mathbf{c}^{\mathrm{T}} \mathbf{H c}-\mathbf{d}^{\mathrm{T}} \mathbf{g}+\mathbf{c}^{\mathrm{T}} \mathbf{G d}
$$

in which

$$
\mathbf{H}=\int_{\Gamma_{e}} \mathbf{Q}^{\mathrm{T}} \mathbf{N} \mathrm{d} \Gamma, \quad \mathbf{G}_{e}=\int_{\Gamma_{e}} \mathbf{Q}^{\mathrm{T}} \tilde{\mathbf{N}} \mathrm{d} \Gamma, \quad \mathbf{g}_{e}=\int_{\Gamma_{q e}} \tilde{\mathbf{N}}^{\mathrm{T}} \bar{q} \mathrm{~d} \Gamma
$$

Minimization of the functional $\Pi_{e}$ with respect to $\mathbf{c}$ and $\mathbf{d}$, respectively, yields

$$
\frac{\partial \Pi_{e}}{\partial \mathbf{c}^{\mathrm{T}}}=-\mathbf{H} \mathbf{c}+\mathbf{G d}=\mathbf{0}, \quad \frac{\partial \Pi_{e}}{\partial \mathbf{d}^{\mathrm{T}}}=\mathbf{G}^{\mathrm{T}} \mathbf{c}-\mathbf{g}=\mathbf{0}
$$

from which the optional relationship between $\mathbf{c}$ and $\mathbf{d}$

$$
\mathbf{c}=\mathbf{H}^{-1} \mathbf{G d}
$$

and the element stiffness equation

$$
\mathbf{G}^{\mathrm{T}} \mathbf{H}^{-1} \mathbf{G d}=\mathbf{g}
$$

are obtained. 


\section{Numerical experiments}

To investigate the effect of packing dimension on effective thermal conductivities of the composites, the influence of packing size ratio $h / l$ of the $\mathrm{RVE}$ is considered here. Also, the material mismatch ratio $k^{\mathrm{I}} / k^{\mathrm{M}}$ between the component phases and the volume fraction $f$ of the inclusion are analysed. Without loss of generality, the thermal conductivity of matrix is assumed to be 1 , and the mismatch ratio is assumed to vary from 0 to 1000 . The mismatch less than unity corresponds to a poorly conducting inclusion case and the minimum value 0 means that the inclusion becomes a hole, while the mismatch greater than unity corresponds to a highly conducting inclusion case and the largest value 1000 is used to approximately model the superconductor, whose thermal conductivity value is extremely higher than that of the popular matrix by several thousand orders. Besides, the ratio $h / l$ of the RVE is assumed to change in the range $[0.5,1.0,1.5]$ with $h=0.5$. Additionally, uniform temperatures of 10 and 0 are imposed at the two opposite sides of the RVE, respectively.

In this study, the HFS-FEM with super hybrid element is extended to perform heat transfer analysis in the RVE for different cases. In the analysis, only one hybrid finite element with 12 quadratic edges and 24 nodes is employed to model the RVE in order to achieve the simple mesh generation and mesh reduction. For example, the element keeps invariant for the variation of the inclusion volume fraction.

Numerical results displayed in Figures 3-5 give the variation of effective thermal conductivities of the composite with the inclusion volume fraction. It is found that the packing size significantly affects the effective thermal conductivities of the composite and causes orthotropy. In contrast to $h / l=0.5$ and 1.5 , the square pattern of the RVE can produce the largest effective thermal conductivities of the composite and the isotropy is also observed. The main reason is that the square pattern can yield the highest volume fraction of the inclusion, i.e. $f=78.5 \%$, whilst, for the case of $h / l=0.5$ and 1.5 , the maximum value of $f$ is just $39.25 \%$ and $52.4 \%$, respectively. Besides, when $k^{\mathrm{I}} / k^{\mathrm{M}}=1$, which means the inclusion and the matrix are the same, either for $h / l=0.5,1.0$ or 1.5 ,
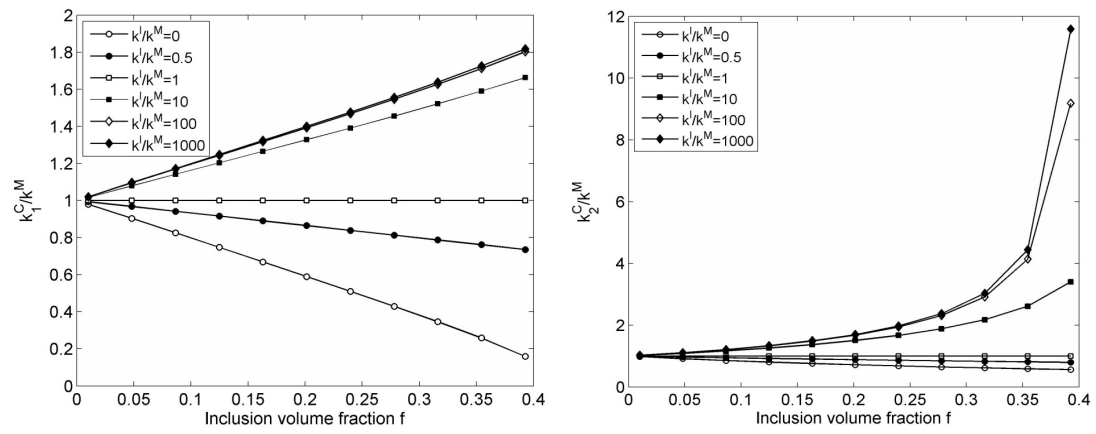

Figure 3: $\quad$ Effective thermal conductivities for the case of $h / l=0.5$. 

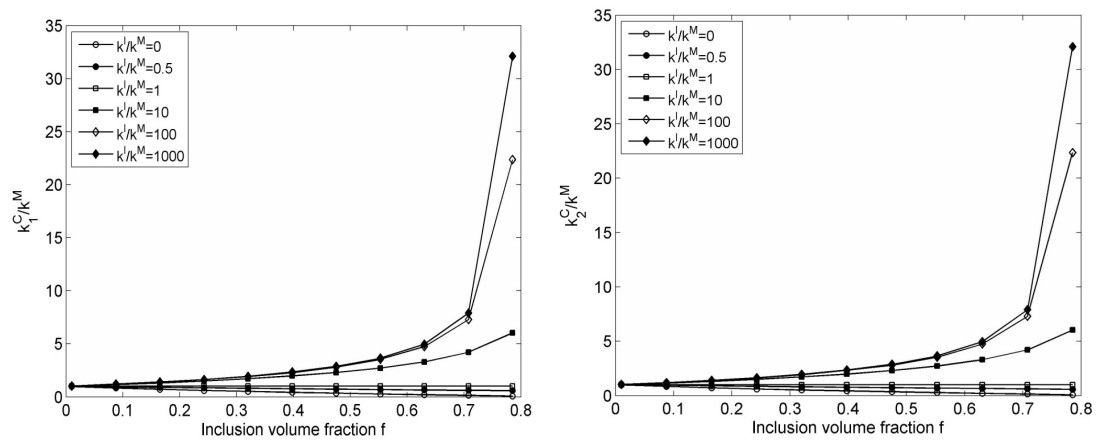

Figure 4: $\quad$ Effective thermal conductivities for the case of $h / l=1.0$.
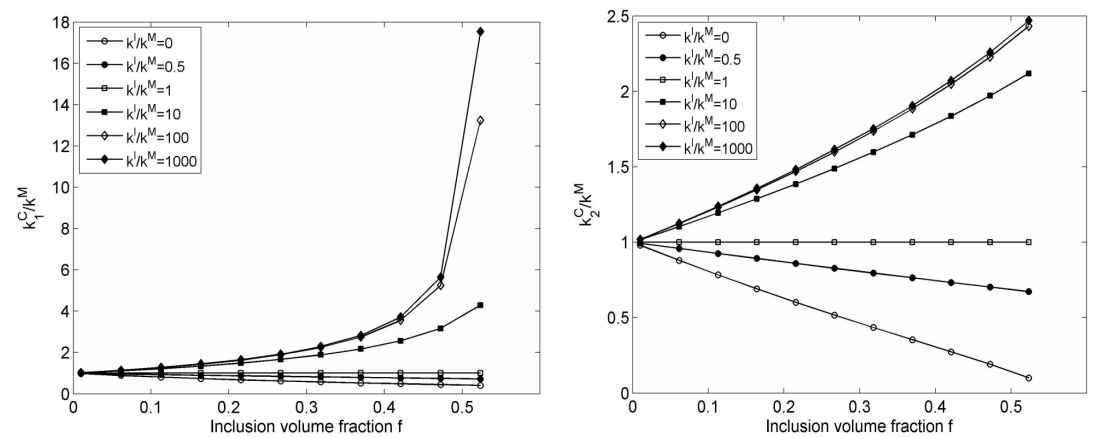

Figure 5: $\quad$ Effective thermal conductivities for the case of $h / l=1.5$.

there are uniform results regardless of the inclusion volume fraction. This phenomenon can be used to verify the present super hybrid element. Moreover, as we expect that the thermal conductivities of the composite become smaller with the increase of $f$ for the case of poorly conducting inclusion, that is $k^{\mathrm{I}} / k^{\mathrm{M}}<1$, whereas they become bigger with the increase of $f$ for the case of highly conducting inclusion, that is $k^{\mathrm{I}} / k^{\mathrm{M}}>1$.

\section{Conclusion}

Numerical results presented in this paper show that the packing dimension of the RVE has a marked influence on the micromechanical behaviour of a composite with periodic double-array inclusions. It is found that the packing size significantly affects the volume fraction of the inclusion and results in evident orthotropy of the composite. Additionally, the super polygonal element developed shows promising advantages in dealing with the heat transfer problems with a complex microstructure and achieving the high modeling efficiency and solution accuracy with relatively low mesh density. The super element boundary can keep geometry invariant when the size of the RVE is 
changed. Based on this feature, an extension of the super element to the case of RVE with clustered inclusions is underway.

\section{Acknowledgements}

The research work is partially supported by the Natural Science Foundation of China (Grant no. 11102059 and 51178165) and the Creative Talents Program of Universities in Henan Province (Grant no. 13HASTIT019).

\section{References}

[1] Islam, M.R., Pramila A., Thermal conductivity of fiber reinforced composites by the FEM. Journal of Composite Materials, 33, pp. 1699$1715,1999$.

[2] Li, S., General unit cells for micromechanical analyses of unidirectional composites. Composites Part A: Applied Science and Manufacturing, 32, pp. 815-826, 2001.

[3] Li, H.Z., Li, S.G., et al., Prediction of effective thermal conductivities of woven fabric composites using unit cells at multiple length scales. Journal of Materials Research, 26, pp. 384-394, 2011.

[4] Goldberg, R.K., Hopkins, D.A., Application of the boundary element method to the micromechanical analysis of composite materials. Computers and Structures, 56, pp. 721-731, 1995.

[5] Qin, Q.H., Micromechanics-BE solution for properties of piezoelectric materials with defects. Engineering Analysis with Boundary Elements, 28, pp. 809-814, 2004

[6] Onyejekwe, O.O., Heat conduction in composite media: a boundary integral approach. Computers and Chemical Engineering, 26, pp. 1621-1632, 2002.

[7] KoŁodziej, J.A., UściŁowska, A., Application of MFS for determination of effective thermal conductivity of unidirectional composites with linearly temperature dependent conductivity of constituents. Engineering Analysis with Boundary Elements, 36, pp. 293-302, 2012.

[8] Wang, H., Qin, Q.H., Hybrid FEM with Fundamental Solutions as trial functions for Heat Conduction Simulation. Acta Mechanica Solida Sinica, 22, pp. 487-498, 2009.

[9] Wang, H., Qin, Q.H., Special fiber elements for thermal analysis of fiberreinforced composites. Engineering Computations, 28, pp. 1079-1097, 2011.

[10] Wang, H., Qin, Q.H., A new special element for stress concentration analysis of a plate with elliptical holes. Acta Mechanica, 223, pp. 1323$1340,2012$.

[11] Wang, H., Qin, Q.H., Fundamental-solution-based hybrid FEM for plane elasticity with special elements. Computational Mechanics, 48, pp. 515$528,2011$. 
[12] Wang, H., Cao, L.L. and Qin, Q.H., Hybrid graded element model for nonlinear functionally graded materials. Mechanics of Advanced Materials and Structures, 19, pp. 590-602, 2012

[13] Wang, H., Qin, Q.H., Boundary integral based graded element for elastic analysis of 2D functionally graded plates. European Journal of MechanicsA/Solids, 33, pp. 12-23, 2012.

[14] Wang, H., Qin, Q.H., and Liang, X.P., Solving the nonlinear Poisson-type problems with F-Trefftz hybrid finite element model. Engineering Analysis with Boundary Elements, 36, pp. 39-46, 2012

[15] Chao, C.K., Shen, M.H., On Bonded Circular Inclusions in Plane Thermoelasticity. Journal of Applied Mechanics, 64, pp. 1000-1004, 1997. 